\title{
STUDIES ON POST HETEROCYCLIZATION OF 1, 3, 4- OXADIAZOLE CONTAINING AZETIDINONES DERIVATIVES AND THEIR BIOLOGICAL STUDIES
}

\author{
J.M. Pandya ${ }^{1, *}$ and J. Mahyavanshi ${ }^{2}$ \\ ${ }^{1}$ Department of Chemistry, Sankalchand Patel University, Visnagar-384315, (Gujarat) India \\ ${ }^{2}$ Department of Chemistry, Sankalchand Patel University, Visnagar-384315, (Gujarat) India \\ *E-mail: jayeshbi1998@yahoo.co.in
}

\begin{abstract}
Synthesis and biological evaluation of novel oxazolidinone derivatives via Schiff's base formation has been studied. Synthesis involves cyclocondensation of novel Schiff's base moieties of previously known 1, 3, 4-oxadiazole with chloro acetyl chloride to give a biologically active compound with good yields. All newly synthesized compounds were confirmed by NMR, IR and elemental study. Biological screening for their antibacterial and antifungal activities have been also studied.
\end{abstract}

Keywords: 1, 3, 4-Oxadiazole, Oxoazetidinone, Cyclocondensation, Antibacterial Activity and Spectral Studies.

(c) RASĀYAN. All rights reserved

\section{INTRODUCTION}

Importance of 1,3,4-oxadiazole class of products have been established and reported for its biological activities like anti-inflammatory, antiviral, antidepressant, antimitotic and antimicrobial activities. ${ }^{1-10}$ Hydrazine derivatives of 1,3,4-oxadiazole moiety also exhibit good biological activities. ${ }^{11-12}$

In combination with 1, 3, 4-oxadiazole moiety, presence of azetidinones group enhances molecular activity in terms of antibacterial, antifungal ${ }^{13}$ antibiotic ${ }^{14}$ activities. Some differently substituted azetidinones derivatives prepared from hydrazine shows medicinal activity like antibacterial, anti fungicidal, analgesic, anti-inflammatory activity. ${ }^{15-19}$ Based on these reports it drawn attention to develop and study a combination of azetidinones and 1,3,4-oxadiazole moieties with the hope of better biological activity and ease of synthesis. Hence novel derivatives of N-(3-chloro-2-aryl-4-oxoazetidin-1-yl)-2-((5phenyl-1,3,4-oxadiazol-2-yl) thio) acetamide (IIIa-h) have been synthesized and studies.

\section{Material and Methods}

\section{EXPERIMENTAL}

All the solvents (99\% pure) were purchased from a commercial supplier, Ahmedabad. All key raw materials were purchased from a commercial vendor. Precoated silica aluminium plates of Merck were used for TLC. Melting points were determined in open capillary tubes and were uncorrected. The IR spectra were recorded in $\mathrm{KBr}$ pellets on a Nicolet 760D spectrometer and ${ }^{1} \mathrm{H}$ NMR spectra were recorded in DMSO with TMS as internal standard on a Brucker spectrometer at $400 \mathrm{MHz}$. LC-MS of selected samples taken on LC-MSD-Trap-SL_01046.

\section{General Procedure}

The compound 2-((5-phenyl-1,3,4-oxadiazol-2-yl)thio)aceto hydrazide (I) prepared as follows: The reaction between 5-Phenyl-1,3,4-oxadiazole-2-thiol with bromoethyl acetate followed by hydrazine hydrate gives 2-((5-phenyl-1,3,4-oxadiazol-2-yl)thio)aceto hydrazide (I). ${ }^{20}$

Rasayan J. Chem., 12(3), 1229-1234(2019)

http://dx.doi.org/10.31788/RJC.2019.1235033

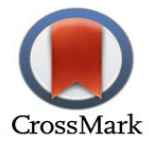


Preparation of N'-aryl-2-((5-phenyl-1, 3, 4-oxadiazol-2-yl) thio) aceto hydrazide (IIa-h)

An equimolecular mixture of 2-((5-phenyl-1, 3, 4-oxadiazol-2-yl) thio) aceto hydrazide (I), (0.01mole) and the aromatic aldehydes $(\mathrm{a}-\mathrm{h})$ in ethanol $(15 \mathrm{ml})$ was refluxed on a water bath for $1-2 \mathrm{hrs}$. The solid separated was collected by filtration, dried and recrystallized from ethanol. The yields, melting points and other characterization data of these compounds are given below.

Preparation of N-(3-chloro-2-aryl-4-oxoazetidin-1-yl)-2-((5-phenyl-1，3，4-oxadiazol-2-yl) thio) acetamide (IIIa-h)

A mixture N'-aryl-2-((5-phenyl-1, 3, 4-oxadiazol-2-yl) thio) aceto hydrazide (IIa-h) (0.002 mole) and triethyl amine (TEA) (0.004 mole) was dissolved in 1, 4-dioxane $(50 \mathrm{ml})$, cooled, and stirred. To this well-stirred cooled solution chloro acetyl chloride ( 0.004 mole) was added drop wise within a period of 30 minutes. The reaction mixture was then stirred for an additional 3 hours and left at room temperature for 48 hours. The resultant mixture was concentrated, cooled, poured into ice-cold water, and then airdried. The product thus obtained was purified by column chromatography over silica gel using $30 \%$ ethyl acetate: $70 \%$ benzene as eluent. Recrystallization from ether/n-hexane gave white powered of $\mathrm{N}-(3-$ chloro-2-aryl-4-oxoazetidin-1-yl)-2-((5-phenyl-1, 3, 4-oxadiazol-2-yl) thio) acetamide (IIIa-h), which was obtained in 53-72\% yield. All the compounds were characterized by analytical and spectral data of the compounds is assigned in Scheme-1.

Yield and Spectroscopic Data of Synthesized Compounds

5-Phenyl-[1, 3, 4] oxadiazol-2-ylsulfanyl)-acetic acid benzylidene-hydrazide IIa

Yield: $85 \%$, solid, m.p.241-243 ${ }^{0} \mathrm{C}$. Anal.Calcd for $\mathrm{C}_{17} \mathrm{H}_{14} \mathrm{~N}_{4} \mathrm{O}_{2} \mathrm{~S}$ : C, 60.34; H, 4.17; N, 16.56; S, 9.48; Found: C, 60.32; H, 4.16; N, 16.55; S, 9.46\%.

5-Phenyl-[1, 3, 4] oxadiazol-2-ylsulfanyl)-acetic acid (4-methoxy-benzylidene)-hydrazide IIb Yield: $79 \%$, solid, m.p.247-249 $9^{0}$. Anal.Calcd for $\mathrm{C}_{18} \mathrm{H}_{16} \mathrm{~N}_{4} \mathrm{O}_{3} \mathrm{~S}$ : C, 58.68; H, 4.38; N, 15.21; S, 8.70; Found: C, 58.67; H, 4.36; N, 15.20; S, 8.69\%.

5-Phenyl-[1, 3, 4] oxadiazol-2-ylsulfanyl)-acetic acid (4-hydroxy-benzylidene)-hydrazide IIc Yield:76\%,solid,m.p.243-245 ${ }^{\circ} \mathrm{C} .{ }^{1} \mathrm{H} \quad$ NMR $\quad(400 \quad$ MHz,DMSO): $\quad \delta \quad 6.8-8.0 \quad(\mathrm{~m}, 10 \mathrm{H}), \quad 8.5$ $(\mathrm{s}, 1 \mathrm{H}), 11.22(\mathrm{~s}, 1 \mathrm{H}), 11.82(\mathrm{~s}, 1 \mathrm{H})$. Anal.Calcd for $\mathrm{C}_{17} \mathrm{H}_{14} \mathrm{~N}_{4} \mathrm{O}_{3} \mathrm{~S}$ :C,57.62; H,3.98; N,15.81; S,9.05.Found: C, 57.60; H, 3.97; N, 15.79; S, 9.04\%.

5-Phenyl-[1, 3, 4] oxadiazol-2-ylsulfanyl)-acetic acid (2-hydroxy-benzylidene)-hydrazide IId Yield: $80 \%$, solid, m.p.256-259 C. Anal.Calcd for $\mathrm{C}_{17} \mathrm{H}_{14} \mathrm{~N}_{4} \mathrm{O}_{3} \mathrm{~S}$ : C, 57.62; H, 3.98; N, 15.81; S, 9.03. Found: C, 57.61; H, 3.96; N, 16.55; S, 9.03\%.

5-Phenyl-[1, 3, 4] oxadiazol-2-ylsulfanyl)-acetic acid (4-methyl-benzylidene)-hydrazide IIe Yield: $74 \%$, solid, m.p.252-255 C. Anal.Calcd for $\mathrm{C}_{18} \mathrm{H}_{16} \mathrm{~N}_{4} \mathrm{O}_{2} \mathrm{~S}$ : C, 61.35; H, 4.58; N, 15.90; S, 9.10. Found: C, 61.34; H, 4.56; N, 15.88; S, 9.08\%.

5-Phenyl-[1, 3, 4] oxadiazol-2-ylsulfanyl)-acetic acid benzo[1,3]dioxol-5-ylmethylene-hydrazide IIf Yield: $78 \%$, solid, m.p.258-261 ${ }^{0}$ C. Anal.Calcd for $\mathrm{C}_{18} \mathrm{H}_{16} \mathrm{~N}_{4} \mathrm{O}_{4} \mathrm{~S}$ : C, 56.54; H, 3.69; N, 14.65; S, 8.39. Found: C, 56.53; H, 3.68; N, 14.63; S, 8.38\%.

\section{5-Phenyl-[1, 3, 4] oxadiazol-2-ylsulfanyl)-acetic acid (4-hydroxy-benzylidene)-hydrazide IIg}

Yield: $77 \%$, solid, m.p.264-266 C.Anal.Calcd for $\mathrm{C}_{17} \mathrm{H}_{14} \mathrm{~N}_{4} \mathrm{O}_{2} \mathrm{~S}$ : C, 56.24; H, 4.20; N, 14.57; S, 8.4. Found: C, 56.22; H, 4.18; N, 14.57; S, 8.33\%.

5-Phenyl-[1, 3, 4] oxadiazol-2-ylsulfanyl)-acetic acid (4-hydroxy-benzylidene)-hydrazide IIh Yield: $75 \%$, solid, m.p.266-269 C. Anal.Calcd for $\mathrm{C}_{21} \mathrm{H}_{22} \mathrm{~N}_{4} \mathrm{O}_{4} \mathrm{~S}$ : C, 59.14; H, 5.20; N, 13.14; S, 7.52. Found: C, 59.32; H, 5.19; N, 13.13; S, 7.50\%. 
3-Chloro-1-[2-oxo-3-(5-phenyl-[1, 3, 4] oxadiazol-2-ylsulfanyl)-propyl]-4-phenyl-azetidin-2-one IIIa Yield:65\%,solid,m.p.227-230 ${ }^{\circ} \mathrm{C} .{ }^{1} \mathrm{H} \quad \mathrm{NMR} \quad(400 \quad \mathrm{MHz}, \mathrm{DMSO}): \quad 86.8-7.5 \quad(\mathrm{~m}, 10 \mathrm{H}), \quad 7.8 \quad(\mathrm{~s}, 1 \mathrm{H})$, 5.2(d,1H),5.3(d,1H), 2.8(s,2H). Anal.Calcd for $\mathrm{C}_{19} \mathrm{H}_{15} \mathrm{ClN}_{4} \mathrm{O}_{3} \mathrm{~S}: \mathrm{C}, 55.01 ; \mathrm{H}, 3.64 ; \mathrm{N}, 13.50, \mathrm{~S}, 7.73$. Found: C, 55.00; H, 3.62; N, 13.48; S, 7.72\%.<smiles>NNC(=O)CSc1nnc(-c2ccccc2)o1</smiles>

2-((5-phenyl-1,3,4-oxadiazol-2-yl)thio)aceto hydrazide (I)

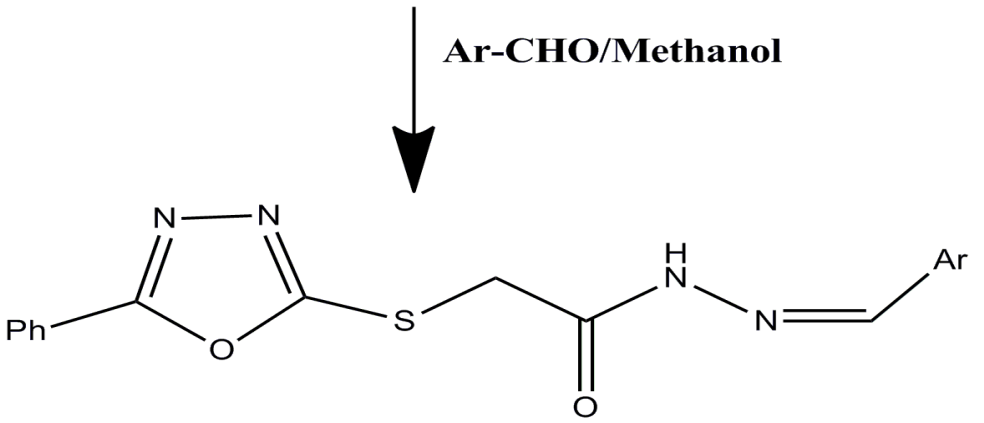

N'-aryl-2-((5-phenyl-1,3,4-oxadiazol-2-yl)thio)aceto hydrazide (Ia-h)

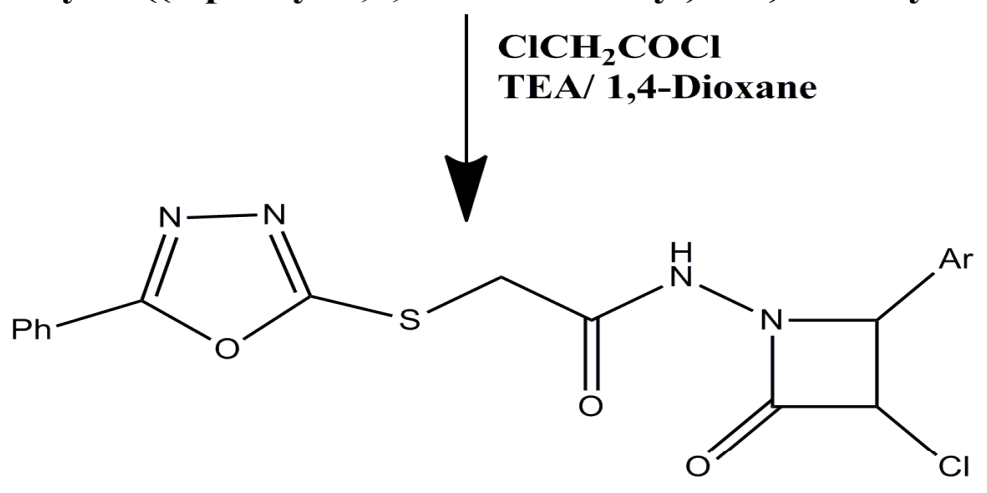

N-(3-chloro-2-aryl-4-oxoazetidin-1-yl)-2-((5-phenyl-1,3,4-oxadiazol-2-yl) thio) acetamide (IIIa-h)
Where, $\mathbf{R}=\left(\right.$ a) $\mathrm{C}_{6} \mathrm{H}_{5}$
(b) $4-\mathrm{OCH}_{3}-\mathrm{C}_{6} \mathrm{H}_{4}$
(c) $4-\mathrm{OH}-\mathrm{C}_{6} \mathrm{H}_{4}$
(d) $2-\mathrm{OH}-\mathrm{C}_{6} \mathrm{H}_{4}$
(e) $4-\mathrm{CH}_{3}-\mathrm{C}_{6} \mathrm{H}_{4}$
(f) $3,4-\mathrm{CH}_{2} \mathrm{O}_{2}-\mathrm{C}_{6} \mathrm{H}_{3}$
(g) $4-\mathrm{OH}-3-\mathrm{OCH}_{3}-\mathrm{C}_{6} \mathrm{H}_{3}$
(h) $3,4-\left(\mathrm{OC}_{2} \mathrm{H}_{5}\right)_{2}-\mathrm{C}_{6} \mathrm{H}_{3}$

Scheme-1

3-Chloro-4-(4-methoxy-phenyl)-1-[2-oxo-3-(5-phenyl-[1, 3, 4] oxadiazol-2-ylsulfanyl)-propyl]azetidin-2-one IIIb

Yield:61\%,solid,m.p.235-238 ${ }^{0} \mathrm{C} .{ }^{1} \mathrm{H} \quad$ NMR $\quad(400 \quad$ MHz,DMSO): $\quad \delta 6.8-7.5 \quad(\mathrm{~m}, 9 \mathrm{H}), \quad 7.8 \quad(\mathrm{~s}, 1 \mathrm{H})$, 5.2(d,1H),5.3(d,1H),3.8(s,2H),2.3(s,3H).Anal.Calcd for $\mathrm{C}_{20} \mathrm{H}_{17} \mathrm{ClN}_{4} \mathrm{O}_{4} \mathrm{~S}: \mathrm{C}, 53.99 ; \mathrm{H}, 3.85 ; \mathrm{N}, 12.59, \mathrm{~S}, 7.21$. Found: C, 53.97; H, 3.83; N, 12.57; S, 7.20\%. 
RASĀYAN J. Chem.

Vol. 12 | No. 3 |1229 - 1234| July - September | 2019

3-Chloro-4-(4-hydroxy-phenyl)-1-[2-oxo-3-(5-phenyl-[1, 3, 4] oxadiazol-2-ylsulfanyl)-propyl]azetidin-2-one IIIc

Yield:58\%,solid,m.p.232-235 ${ }^{\circ} \mathrm{C} .{ }^{1} \mathrm{H} \quad \mathrm{NMR} \quad(400 \quad \mathrm{MHz}, \mathrm{DMSO}): \quad \delta 6.8-7.5 \quad(\mathrm{~m}, 9 \mathrm{H}), \quad 7.8 \quad(\mathrm{~s}, 1 \mathrm{H})$, 5.2(d,1H),5.3(d,1H),3.8(s,2H),11.22(s,1H).Anal.Calcd for $\quad \mathrm{C}_{19} \mathrm{H}_{15} \mathrm{ClN}_{4} \mathrm{O}_{4} \mathrm{~S}$ : C,52.96;H,3.51;N,13.00,S,7.44. Found: C, 52.94; H, 3.50; N, 12.98; S, 7.43\%.

3-Chloro-4-(2-hydroxy-phenyl)-1-[2-oxo-3-(5-phenyl-[1, 3, 4] oxadiazol-2-ylsulfanyl)-propyl]azetidin-2-one IIId

Yield:60\%,solid,m.p.246-249 ${ }^{0} \mathrm{C} .{ }^{1} \mathrm{H} \quad \mathrm{NMR} \quad(400 \quad \mathrm{MHz}, \mathrm{DMSO}): \quad \delta 6.8-7.5 \quad(\mathrm{~m}, 9 \mathrm{H}), \quad 7.8 \quad(\mathrm{~s}, 1 \mathrm{H})$, 5.2(d,1H),5.3(d,1H),3.8(s,2H),2.3(s,3H)11.22(s,1H).Anal.Calcd for $\quad \mathrm{C}_{19} \mathrm{H}_{15} \mathrm{ClN}_{4} \mathrm{O}_{4} \mathrm{~S}$ : C,52.96;H,3.51;N,13.00,S,7.44. Found: C, 52.95; H, 3.49; N, 12.99; S, 7.42\%.

3-Chloro-1-[2-oxo-3-(5-phenyl-[1, 3, 4] oxadiazol-2-ylsulfanyl)-propyl]-4-p-tolyl-azetidin-2-one IIIe Yield:53\%,solid,m.p.238-242 ${ }^{0} \mathrm{C} .{ }^{1} \mathrm{H} \quad$ NMR $\quad(400 \quad$ MHz,DMSO): $\quad \delta 6.8-7.5 \quad(\mathrm{~m}, 9 \mathrm{H}), \quad 7.8 \quad(\mathrm{~s}, 1 \mathrm{H})$, 5.2(d,1H),5.3(d,1H)3.8(s,2H),2.3(s,3H)..Anal.Calcd for $\mathrm{C}_{20} \mathrm{H}_{17} \mathrm{ClN}_{4} \mathrm{O}_{3} \mathrm{~S}: \mathrm{C}, 56.01 ; \mathrm{H}, 4.00 ; \mathrm{N}, 13.06, \mathrm{~S}$, 7.48. Found: C, 56.00; H, 3.99; N, 13.04; S, 7.47\%.

4-Benzo [1, 3] dioxol-5-yl-3-chloro-1-[2-oxo-3-(5-phenyl-[1, 3, 4] oxadiazol-2-ylsulfanyl)-propyl]azetidin-2-one IIIf

Yield:55\%,solid,m.p.240-243 ${ }^{0} \mathrm{C} .{ }^{1} \mathrm{H} \quad$ NMR $\quad(400 \quad$ MHz,DMSO): $\quad \delta 6.8-7.5 \quad(\mathrm{~m}, 8 \mathrm{H}), \quad 7.8$ $(\mathrm{s}, 1 \mathrm{H}), 6.08(\mathrm{~s}, 2 \mathrm{H}), 5.2(\mathrm{~d}, 1 \mathrm{H}), 5.3(\mathrm{~d}, 1 \mathrm{H}) 3.3 .8(\mathrm{~s}, 2 \mathrm{H})$. Anal.Calcd for $\quad \mathrm{C}_{20} \mathrm{H}_{15} \mathrm{ClN}_{4} \mathrm{O}_{5} \mathrm{~S}$ : C,52.35;H,3.29;N,12.21,S,6.99. Found: C, 52.34; H, 3.27; N, 12.20; S, 6.98\%.

3-Chloro-4-(4-hydroxy-3-methoxy-phenyl)-1-[2-oxo-3-(5-phenyl-[1，3，4] oxadiazol-2-ylsulfanyl)propyl]-azetidin-2-one IIIg

Yield:60\%,solid,m.p.253-256 ${ }^{0}$ C. ${ }^{1} \mathrm{H}$ NMR (400 MHz,DMSO): $86.8-7.5(\mathrm{~m}, 8 \mathrm{H}), 7.8(\mathrm{~s}, 1 \mathrm{H}), 5.2(\mathrm{~d}, 1 \mathrm{H}), 5.3$ $(\mathrm{d}, 1 \mathrm{H}) 11.22(\mathrm{~s}, 1 \mathrm{H}), 3.8(\mathrm{~s}, 2 \mathrm{H}), 3.2(\mathrm{~s}, 3 \mathrm{H})$. Anal.Calcd for $\mathrm{C}_{20} \mathrm{H}_{17} \mathrm{ClN}_{4} \mathrm{O}_{5} \mathrm{~S}:$ C, 52.12; $\mathrm{H}, 3.72 ; \mathrm{N}, 12.16, \mathrm{~S}, 6.96$. Found: C, 52.11; H3.71; N, 12.14; S, 6.95\%.

3-Chloro-4-(3, 4-diethoxy-phenyl)-1-[2-oxo-3-(5-phenyl-[1, 3, 4] oxadiazol-2-ylsulfanyl)-propyl]azetidin-2-one IIIh

Yield:52\%,solid,m.p.257-259 ${ }^{0}$ C. ${ }^{1} \mathrm{H}$ NMR (400 MHz,DMSO): $\delta 6.8-7.5(\mathrm{~m}, 8 \mathrm{H}), 7.8(\mathrm{~s}, 1 \mathrm{H}), 5.2(\mathrm{~d}, 1 \mathrm{H}), 5.3$ $(\mathrm{d}, 1 \mathrm{H}), 4.1(\mathrm{q}, 4 \mathrm{H}), 2.9(\mathrm{~s}, 2 \mathrm{H}), 1.3(\mathrm{t}, 6 \mathrm{H})$. Anal.Calcd for $\mathrm{C}_{23} \mathrm{H}_{23} \mathrm{ClN}_{4} \mathrm{O}_{5} \mathrm{~S}$ : C,54.92; $\mathrm{H}, 4.61 ; \mathrm{N}, 11.14, \mathrm{~S}, 6.38$. Found: C, 54.90; H, 4.60; N, 11.12; S, 6.36\%.

\section{Antibacterial Activities}

Antibacterial activities of all the compounds were studied against gram-positive bacteria (Staphylococcus aureus and Bacillus subtilis) and gram-negative bacteria (E.coli, and klebsiella promioe) at a concentration of $50 \mu \mathrm{g} / \mathrm{ml}$ by agar cup plate method. Methanol system was used as a control in this method. Under similar condition using tetracycline as a standard for comparison carried out control experiment. The area of inhibition of zone measured in $\mathrm{mm}$. Compound $3 \mathrm{c}$, $3 \mathrm{f}$ and $3 \mathrm{~g}$ were found more active against the above microbes. Other compounds found to be less or moderate active than tetracycline (Table -1).

Table-1: Antibacterial Activity of Compounds (3a-h)

\begin{tabular}{c|c|c|c|c}
\hline \multirow{2}{*}{ Compounds } & \multicolumn{2}{|c|}{ Gram -Ve } & \multicolumn{2}{c}{ Gram +Ve } \\
\cline { 2 - 5 } & E.coli & $\begin{array}{c}\text { Klebsiella } \\
\text { promioe }\end{array}$ & $\begin{array}{c}\text { Bacillus } \\
\text { subtilis }\end{array}$ & 55 \\
\hline 3a & 65 & 51 & 61 & 60 \\
\hline $3 \mathrm{~b}$ & 54 & 62 & 69 & 65 \\
\hline $3 \mathrm{c}$ & 68 & 53 & 59 & 54 \\
\hline $3 \mathrm{~d}$ & 59 & 61 & 51 & 54 \\
\hline $3 \mathrm{e}$ & 65 & 64 & & \\
\hline
\end{tabular}


RASĀYAN J. Chem.

Vol. 12 | No. 3 |1229 - 1234| July - September | 2019

\begin{tabular}{c|l|l|l|l}
\hline $3 \mathrm{f}$ & 63 & 58 & 72 & 70 \\
\hline $3 \mathrm{~g}$ & 59 & 61 & 65 & 71 \\
\hline $3 \mathrm{~h}$ & 60 & 43 & 63 & 68 \\
\hline Tetracycline & 78 & 86 & 79 & 67 \\
\hline
\end{tabular}

\section{Antifungal Activities}

The fungicidal activity of all the compounds was studied at $1000 \mathrm{ppm}$ concentration in vitro. Plant pathogenic organisms used were (Nigrospora Sp, Aspergillus niger, Botrydepladia thiobromine, and Rhizopus nigricum, Fusarium oxyporium.) The antifungal activity of all the compounds (3a-h) was measured on each of these plant pathogenic strains on a potato dextrose agar (PDA) medium. Such a PDA medium contained potato $200 \mathrm{gm}$, dextrose $20 \mathrm{gm}$, agar $20 \mathrm{gm}$ and water one liter. Five days old cultures were employed. The compounds to be tested were suspended (1000ppm) in a PDA medium and autoclaved at $120^{\circ} \mathrm{C}$ for $15 \mathrm{~min}$. at $15 \mathrm{~atm}$.pressure. This medium was poured into sterile Petri plates and the organisms were inoculated after cooling the Petri plates. The percentage inhibition for fungi was calculated after five days using the formula given below:

Where, $X=$ Area of the colony in the control plate

Percentage of inhibition $=100(\mathrm{X}-\mathrm{Y}) / \mathrm{X}$

$\mathrm{Y}=$ Area of the colony in the test plate

The fungicidal activity displayed by various compounds (3a-h) is shown in Table-2.

Table-2: Antifungal Activity of Compounds (3a-h)

\begin{tabular}{|c|c|c|c|c|c|}
\hline \multicolumn{6}{|c|}{ Zone of Inhibition at $1000 \mathrm{ppm}(\%)$} \\
\hline Compounds & $\begin{array}{c}\text { Aspergillus } \\
\text { niger }\end{array}$ & $\begin{array}{c}\text { Botrydepladia } \\
\text { Thiobromine }\end{array}$ & $\begin{array}{l}\text { Fusarium } \\
\text { oxyporium }\end{array}$ & Nigrospora Sp. & $\begin{array}{l}\text { Rhizopus } \\
\text { Nigricum }\end{array}$ \\
\hline $3 a$ & 60 & 61 & 68 & 60 & 50 \\
\hline $3 \mathrm{~b}$ & 55 & 62 & 65 & 59 & 64 \\
\hline $3 \mathrm{c}$ & 58 & 64 & 68 & 55 & 60 \\
\hline $3 \mathrm{~d}$ & 44 & 62 & 63 & 64 & 71 \\
\hline $3 \mathrm{e}$ & 63 & 63 & 59 & 59 & 68 \\
\hline $3 f$ & 52 & 64 & 62 & 63 & 60 \\
\hline $3 g$ & 58 & 70 & 58 & 56 & 53 \\
\hline $3 \mathrm{~h}$ & 64 & 68 & 62 & 61 & 70 \\
\hline
\end{tabular}

\section{RESULTS AND DISCUSSION}

It was observed that 2-((5-phenyl-1,3,4-oxadiazol-2-yl)thio)aceto hydrazide (I) on condensation with aromatic aldehydes to yield N'-aryl-2-((5-phenyl-1,3,4-oxadiazol-2-yl)thio)aceto hydrazide (IIa-h).The structures of (IIa-h) were confirmed by elemental analysis and IR spectra showing absorption band at $1625-1642(\mathrm{C}=\mathrm{N}), 3370-3385(\mathrm{~N}-\mathrm{H}), 3024-3085 \mathrm{~cm}^{-1}$ (C-H, of Ar.), 1675-1700 $\mathrm{cm}^{-1}(-\mathrm{CO}), 2900,1350 \mathrm{~cm}^{-}$

${ }^{1}\left(-\mathrm{CH}_{2},-\mathrm{CH}_{3}\right), 1181(\mathrm{C}-\mathrm{S}), 1630(\mathrm{C}=\mathrm{N}$ ring $), 755(\mathrm{C}-\mathrm{O}-\mathrm{C}$ ring $), 2825-2860 \mathrm{~cm}^{-1}\left(-\mathrm{OCH}_{3}\right), 3400-3470 \mathrm{~cm}^{-1}(-$ $\mathrm{OH})$. The $\mathrm{C}, \mathrm{H}, \mathrm{N}$ analysis of all compounds is presented above.

The Cyclocondensation of (IIa-h) with chloroacetylchloride resulted in formation of N-(3-chloro-2-aryl-4oxoazetidin-1-yl)-2-((5-phenyl-1, 3, 4-oxadiazol-2-yl) thio) acetamide (IIIa-h). The structures assigned to (IIIa-h) were supported by the elemental analysis and IR spectra showing absorption bands at 1700-1685

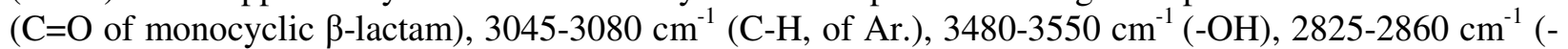
$\left.\mathrm{OCH}_{3}\right), 2950,1370 \mathrm{~cm}^{-1}\left(-\mathrm{CH}_{2},-\mathrm{CH}_{3}\right), 1182(\mathrm{C}-\mathrm{S}), 1591(\mathrm{C}=\mathrm{N}$ ring),764(C-O-C ring). The $\mathrm{C}, \mathrm{H}, \mathrm{N}$ analysis and ${ }^{1} \mathrm{H}-\mathrm{NMR}$ data of all compounds are presented above.

The examination of data reveals that the elemental contents are consistency with the predicted structure shown in scheme-1. The IR data are also direct for the assignment of the predicted structure. The final structure of all compounds is confirmed by LC-MS data of selected samples. The LC-MS of samples $3 b$ and $3 \mathrm{e}$ give the molecular ion peak $(\mathrm{m} / \mathrm{z})$ at 444 and 428 respectively. These values correspond to their molecular weight. 
RASĀYAN J. Chem.

Vol. 12 | No. 3 |1229 - 1234| July - September | 2019

\section{ACKNOWLEDGMENT}

The authors are thankful to Head, Department of Chemistry, Smt S.S. Patel Nootan Science and Commerce CollegeVisnagar for providing laboratory facilities.

\section{REFERENCES}

1. Z. Li, P. Zhan, X. Liu, Mini-Reviews in Medicinal Chemistry, 11, 13(2011),DOI: 10.2174/138955711797655407

2. S. Bajaj, P. Roy, J. Singh, Indian Journal of Pharmaceutical Education and Research, 51(2),260(2017), DOI:10.5530/ijper.51.2.31

3. N. Polkam, B. Kummari, P. Rayam,U. Brahma,V.Modi Naidu and J. S. Anireddy, Chemistry Select, 2(20), 5492(2017), DOI:10.1002/slct.2017011101

4. S.A.Khan, P. Ahuja and A. Husain, Chinese Chemical Society,64(8), 918(2017), DOI: 10.1002/jccs.201600199

5. A. Parameshwar, V. Selvam, Majid Ghashang and S. Guhanathan, Oriental Journal of Chemistry, 33(3), 1502(2017), DOI:10.13005/ojc/330355

6. V. Nieddu, G. Pinna, I.Marchesi, and G. Murineddu, J. Med. Chem., 59 (23), 10451(2016), DOI:10.1021/acs.jmedchem.6b00468

7. A. Borsoi, M. Coldeira, K. Pissinate and D.S. Santos, An International Journal for Rapid Communication of Synthetic Organic Chemistry, 47(14), 1319 (2017), DOI:10.1080/00397911.2017.1324626

8. G. Dayakar and A. Jeyanthi, Der Pharma Chemica, 5(6), 341(2013)

9. E. Palaska, G. Sahin, P. Kelicen, N.T. Durlu and G. Altinok, IL Farmaco, 57, 101(2002), DOI: $10.1016 / \mathrm{S} 0014-827 \mathrm{X}(01) 01176-4$

10. M. Kouhkan, F. Karimi,A. Souldozi and J. Rashedi, Int. J. Adv. Res, 5(5), 1468(2017), DOI: $10.21474 / \mathrm{IJAR} 01 / 4269$

11. P. Patraoa , A. M. A. Khadera, B. Kallurayaa and Vinayachandra, Der Pharma Chemica, 5(2), 24(2013).

12. Al- Mawsawi LQ, R. Dayam, L. Taheri, M. Witvrouw, N. Neamati, Bioorg. Med. Chem. Lett. 17(23), 6472(2007), DOI:10.1016/j.bmcl.2007.09.102

13. V. V. Mulwad, B. P. Choudhari, Indian J. heterocyclic Chem, 12, 197(2003)

14. K.Akiba,M.Wada,Chem.Abstr, 111, 96964b (1989).

15. E. Gursoy, N. Guzeldemirci-Ulusoy, Eur. J. Med. Chem, 42, 320(2007), DOI:10.1016/j.ejmech.2006.10.012

16. Y.Janin, Bioorg.Med.Chem. 15, 2479(2007) DOI: 10.1016/j.bmc.2007.01.030

17. K. K. Murahari, H. R. Gangadasu, T. Suresh, C. C. Kalyan, R. Kaur, P. Burange, J.Ghogare, V.Mokale, M.Raut, Bioorg.Med. Chem, 15, 3997(2007), DOI:10.1016/j.bmc.2007.04.003

18. D. Sriram, P. Yogeeswari, K. Madhu, Bioorg. Med. Chem. Lett, 16(4), 876(2006), DOI:10.1016/j.bmcl.2005.11.004

19. M. R. Rao, N. Devanna and K. B. Chandrasekhar, Asian J. Chem, 20, 1402(2008)

20. K.K.Jha, Y. Kumar and M. Shaharyar, Asian Journal of Chemistry, 21(9) , 7403(2009)

[RJC-5033/2019] 